\title{
Experience in the management of a severe burn patient with multiple complications
}

\author{
Xu-sheng $L^{1 *}$, Ji-chao $Y^{2}$, Hua-bing $Q^{2}$, Weidong $G^{2}$, Hong $Y^{2}$, Qi-zhi $L^{3}$, Li-ju $T^{3}$ and Xian-hui $H^{3}$ \\ ${ }^{1}$ Department of Burns, The First Affiliated Hospital Sun Yat-sen University, Guangzhou, Guangdong, PR China \\ ${ }^{2}$ Department of Burns, The Affiliated HouJie Hospital of Guang Dong Medical College, Dongguan, Guangdong, PR China \\ ${ }^{3}$ Burn Institute, The First Affiliated Hospital of Army Medical University, Chongqing, PR China
}

\section{Case report}

The patient is a male worker, 33 yrs. Old, Injured by hot steel residuals. He was admitted to the local hospital at $1 \mathrm{PBH}$ with the diagnosis of burns by hot steel residuals with TBSA of $95 \%$ in which $10 \%$ deep II $^{\circ}$ and $85 \% \mathrm{III}^{\circ}$ throughout the whole-body surface. Tracheostomy was done in the local hospital with the initiation of venous fluid resuscitation and the escharotomy on the trunk and four extremities. He was transferred to our hospital at 58 PBHs. During the hospitalization, the patient suffered from many postburn complications, such as pulmonary failure, hypovolemic shock, severe systemic infection and sepsis, thrombosis in the deep and shallow venous blood vessels in both legs, stress diabetics mellitus, repeated outbreaks of acute pancreatitis. Comprehensive management was employed in the treatment of these complications in addition to major burn and inhalation injury with satisfactory results.

Experience and lessons in the management of this severe burn patient

Maintenance of the hemodynamics in case of hypovolemic shock and during peri-operative period

The patient was admitted to the local hospital immediately at 0.5 to $1 \mathrm{PBH}$ with the establishment of intravenous route way for fast and abundant fluid infusion. There was $19000 \mathrm{ml}$ fluid infused within the $1 \mathrm{st}$ $24 \mathrm{PBHs}$ in which the plasma occupied for $4000 \mathrm{ml}$. And $9000 \mathrm{ml}$ fluid was infused intravenously in the 2nd $24 \mathrm{PBHs}$.

The patient received invasive hemodynamic monitoring after his admission to the central hospital. Fluid resuscitation and anti-shock treatment were carried out under the monitoring, which exhibited obvious advantages in the correction of burn shock and during the operation [1]. But there was much more fluid infused during the shock period in this patient than the formula indication, which needs to be explored for advantages and disadvantages.

\section{Management of the III degree burn wound in this patient}

There was $85 \%$ TBSA of III degree burn wound in this patient.

Escharotomy was performed at $4 \mathrm{PBHs}$ so as to release the tension of the eschar. The 1st time of escharectomy and micro-skin grafting was carried out on the 6 PBD. And 15 times of wound debridement and skin grafting were performed thereafter with intense wound management around the operation.

Small pieces of alloskin or artificial skin were covered onto the burn wound during the preparation of the wound due to the shortage of autoskin. Sensitive antibiotics were selected and applied onto the patient's wound according to the results of bacterial culture of the wound samples.

\section{Management of severe inhalation injury and pulmonary failure}

Tracheostomy was performed on $6 \mathrm{PBH}$ followed by tracheal intubation. Oxygen supply, sputum aspiration, aerosol inhalation and airway lavage were performed via the intubation.

Mechanical ventilation in synchronized respiratory support was employed when there was hypoxia and pulmonary failure. Interval blood gas analysis was employed for the monitoring and the modulation of oxygen inhalation and respirator index. Ambroxol hydrochloride was infused intravenously as expectorant medication.

\section{Severe systemic infection}

The infection exhibited fever $\left(39 \sim 40^{\circ} \mathrm{C}\right)$, dottiness, increase of WBC, red and swelling burn wound with pain and hemorrhagic tendency, increased exudation from wound granulation tissue and increased burn wound pus.

Bacterial examination and drug sensitive test were performed with the samples from burn wound and the blood stream. High quality and broad-spectrum antibiotics (e.g. Vancomycin, Teicoplanin, Imipenem, Cefepime, Itraconazole, etc) were applied as early as possible with the assistance of the transfusion of fresh blood or plasma and operational debridement and skin grafting for wound closure.

\section{Thrombosis in both legs}

Catheterization via both sides of femoral vein was performed since the 1st day after admission, so as to guarantee fluid infusion. The right lower leg swelling was found on the 46 PBDs. DSA and type $\mathrm{B}$ sonography was performed to check the blood vessels in the legs. Intravenous thrombosis was found in the venous vessels in the right leg. Thereafter, the thrombosis developed in both legs with swelling and exudation in both legs. It was also found that the epithelia grew slowly.

Therefore, the following procedures were employed:

1. Elevation of both legs. Stop all catheterization via the legs. All the fluid infusion route was changed to arms or head and cervical venous blood vessels;

${ }^{\star}$ Correspondence to: Xu-sheng L, Department of Burns, The First Affiliated Hospital Sun Yat-sen University, Guangzhou, Guangdong, PR China, Tel: +86 13925013885; E-mail: liuxusheng7984@126.com

Received: April 19, 2018; Accepted: May 14, 2018; Published: May 17, 2018 
2. Low molecular dextran (500ml per time), intravenously;

3. Urokinase was intravenously infused once every day in dose of 0.1 MIU dissolved in $250 \mathrm{ml}$ of $5 \%$ GS (glucose solution);

4. Oral taking of enteric coating Aspirin $(25 \mathrm{mg}$ per time and $(25 \mathrm{mg}$ per time and 3 times a day);

5. Urokinase $(0.3 \mathrm{MIU})$ was infused to the thrombotic vessels once a day for 9 days;

6. Normal saline $(0.9 \% \mathrm{NaCl})$ with low molecular heparin sodium $(0.8 \mathrm{ml})$ was infused once a day.

After 45 days of treatment with the above procedures, the swelling state of the both legs were corrected. It was indicated by color sonography that the deep and shallow venous blood flow became continuous with the thrombi organization along the inner wall of vascular lumen.

The formation of thrombosis in both legs was closely related to a number of procedures, such as early venous excide and repeated puncture of both femoral blood vessels and long-lasted bedrid state, as well as the application of hemostatic reagents for the control of wound bleeding. The similar cases were once reported by Chen [2] et al that the development of postburn DVT (deep venous thrombosis) in 26 cases of burn patients were closely related to venotomy, long-term venous transfusion and long-term bedrid state, etc. The successful management procedures included diagnosis with type B sonography and DSA, as well as the application of thrombolytic drugs (urokinase), anti-coagulant (low molecular heparin) and low molecular dextran. In addition, oral taking of aspirin, adequate activity and avoiding catheterization of both legs were necessary.

\section{Repeated outbreaks of acute pancreatitis}

Windy and vomiting but not abdominal pain appeared after the operation on 137 PBDs and repeated for several times thereafter. Pancreatitis was suggested on 141 PBDs. Blood amylase was checked as $677.0 \mathrm{IU} / \mathrm{L}$ while the urine amylase was $3832.4 \mathrm{IU} / \mathrm{L}$. The diagnosis was confirmed and fasting of water and food was employed immediately after that. By the way, the inhibition of the secretion of gastric acid and pancreas and antibiotic reagents were applied. In addition, 654-2 (20mg, VD, once per day) and losec (40mg, IV, twice a day).

Three mg of Stilamin was added to $50 \mathrm{ml}$ of normal saline (NS) and which was injected intravenously by venous pump once per 12 hours. $0.3 \mathrm{M}$ IU of Ulinastatin was added to $100 \mathrm{ml}$ for intravenous infusion twice a day. Hankang (Somatostatin for injection) $3 \mathrm{mg}$ was added to $50 \mathrm{ml}$ of NS, venous pump injection once a day. Aperiodic detection of blood and urine samples were carried out.

The pancreatic images were observed by type B sonography. During the hospitalization, accidental food intake would induce the repeated outbreak of pancreatitis with blood amylase increasing to 1711.8
IU/L. Fast and medication with the above drugs was employed for the treatment. At last, the blood amylase recovered to normal range with the disappearing of clinical signs of pancreatitis on 139 days after the occurring. The patient's appetite and oral food recovered gradually.

The preliminary out-breaking of the pancreatitis exhibited ordinary symptoms of gastroenteritis, which was easily misunderstood as that due to filthy food. Lin [3] et al once reported that ventosity was one of the typical and common signs of the patients during the early stage of the attack.

\section{Pay attention to systemic nutrition support during the whole process of treatment}

The overall per day requirement of fluid, colloid, calory and protein of the patient was calculated since the 3rd PBDs. And the nutrition support was applied which included three litres bag, blood ware for parenteral nutrition.

Oral nutrients such as Fresubin MCT 750, Supportan, nutrison were applied when enteral function was normal. Growth hormone (12 16 IU) was injected subcutaneously per night. The balance of water, electrolytes and acid-base was maintained, so as to keep the hemoglobin more than $100 \mathrm{~g} / \mathrm{L}$ and the total protein more than $60 \mathrm{~g} / \mathrm{L}$. Enough calory and protein should be provided, and the trauma repair could be guaranteed.

\section{Experience concluded in the management}

1. Early postburn fluid resuscitation for burn shock and escharotomy;

2. Early escharectormy and skin grafting

3. Coverage of the granulation wound with alloskin before autoskin, so as to reduce nutrition loss and to avoid infection and to prepare for the operation;

4. Active nutrition support and the application of growth hormone;

5. Intensive peri-operative care, especially the maintaining of temperature and hemodynamics (invasive monitoring).

6. Early diagnosis and management of various complications;

7. Application of highly-effective antibiotics (for G+, G- and fungi) in time and in full dose during the early, peri-operative and infection risk periods.

\section{References}

1. Yi-ping Z (2001) The transport of burnt victims. In "Li Ao's Burns Surgery", Li Ao editor-in-chief, Shanghai, Shanghai Science and Technology Press (Chinese).

2. Yu-ming C, Zhong-min X, Geng-qi W (2000) Burns complicated by the formation of deep venous thrombosis-prevention, management and analysis of mechanism in 26 patients. Modern Medicine and Health 16:411-412.

3. Yuan L, Xin-sen L, Run-xiu W (1996) Three cases of acute pancreatitis complicated in major burn patients. Chinese Journal of Plastic and Burn Surgery 12: 460.

Copyright: $\odot 2018$ Xu-sheng L. This is an open-access article distributed under the terms of the Creative Commons Attribution License, which permits unrestricted use, distribution, and reproduction in any medium, provided the original author and source are credited. 